\title{
Innovative strategies in metastatic gastric cancer: a short review
}

\author{
Hannah Christina Puhr (D) - Aysegul Ilhan-Mutlu (D)
}

Received: 6 July 2021 / Accepted: 22 September 2021 / Published online: 19 October 2021

(C) The Author(s) 2021

\begin{abstract}
Summary Recent innovative advances, especially concerning immunotherapeutic agents and targeted therapies, have changed the face of modern oncology. The year 2020 represents a milestone in the treatment of gastroesophageal cancer because several trials showed promising survival benefits, at least for a specific subgroup of patients. Not only immunotherapeutic agents, but also targeted therapies seem to be beneficial, particularly when the target is well defined and the threshold value is selected appropriately. Thus, many new innovative treatment strategies are underway and might lead to a further paradigm change in the therapy of patients with advanced gastric tumors. This review gives a concise overview of these new therapeutic options and recently approved strategies as well as ongoing studies.
\end{abstract}

Keywords Gastroesophageal cancer · Gastric cancer • Immunotherapy · Targeted therapy · Upper gastrointestinal cancer · Advanced cancer

$\begin{array}{ll}\text { Abbreviations } \\ \text { CI } & \text { Confidence interval } \\ \text { CPS } & \text { Combined Positive Score } \\ \text { DoR } & \text { Duration of response } \\ \text { EMA } & \text { European Medicines Agency } \\ \text { FDA } & \text { Food and Drug Administration } \\ \text { FGFR2b } & \text { Fibroblast growth factor receptor 2b } \\ \text { GEJ } & \text { Gastroesophageal junction } \\ \text { HER2 } & \text { Human epidermal growth factor receptor } 2 \\ \text { HR } & \text { Hazard ratio }\end{array}$

H. C. Puhr, MD · A. Ilhan-Mutlu, MD PhD ( $ه)$

Department of Medicine I, Division of Oncology, Medical University of Vienna, Vienna, Austria

aysegul.ilhan@meduniwien.ac.at

H. C. Puhr, MD

hannah.puhr@meduniwien.ac.at

\author{
MSI-H High microsatellite instability \\ n.r. $\quad$ Not reached \\ ORR Overall response rate \\ OS Overall survival \\ PD-(L)1 Programmed cell death receptor (ligand) 1 \\ PFS Progression-free survival \\ TMB-H High tumor mutational burden
}

\section{Introduction}

Gastric cancer is a major contributor to global disease burden and, thus, new treatment options are desperately needed to improve the outcome of patients suffering from this devastating disease [1]. Although advances in immunotherapy as well as targeted therapy at the beginning of the 21st century have led to major breakthroughs in various types of cancer, these high expectations could only recently be met in trials concerning gastric cancer [2]. Thus, new innovative targets and drugs are underway to improve the care of patients with gastric cancer. The aim of this minireview is to concisely highlight some promising new treatment approaches of metastatic gastric cancer and their clinical relevance. Trials, which showed clinical and statistical relevant data leading to approval of new therapeutic strategies by appropriate authorities for advanced gastric cancer patients in recent years, are listed in Table 1.

\section{Immunotherapy for gastric cancer}

\section{Current treatment landscape with immunotherapeutic agents}

So far, the most promising immunotherapeutic target in gastric cancer patients was the programmed cell death receptor 1 (PD-1)/programmed cell death ligand 1 (PD-L1) checkpoint axis [3]. Although some 


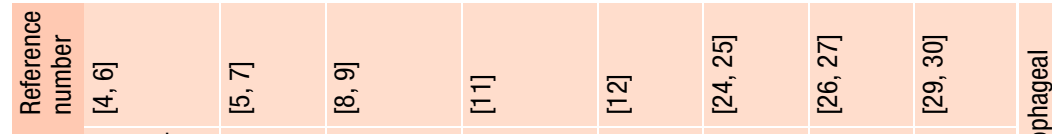

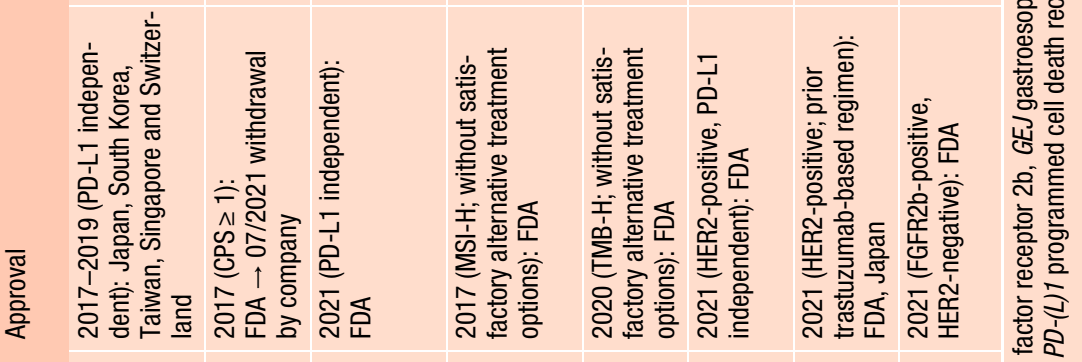

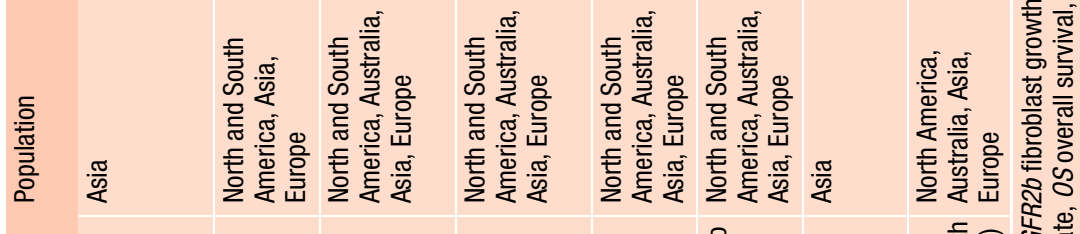

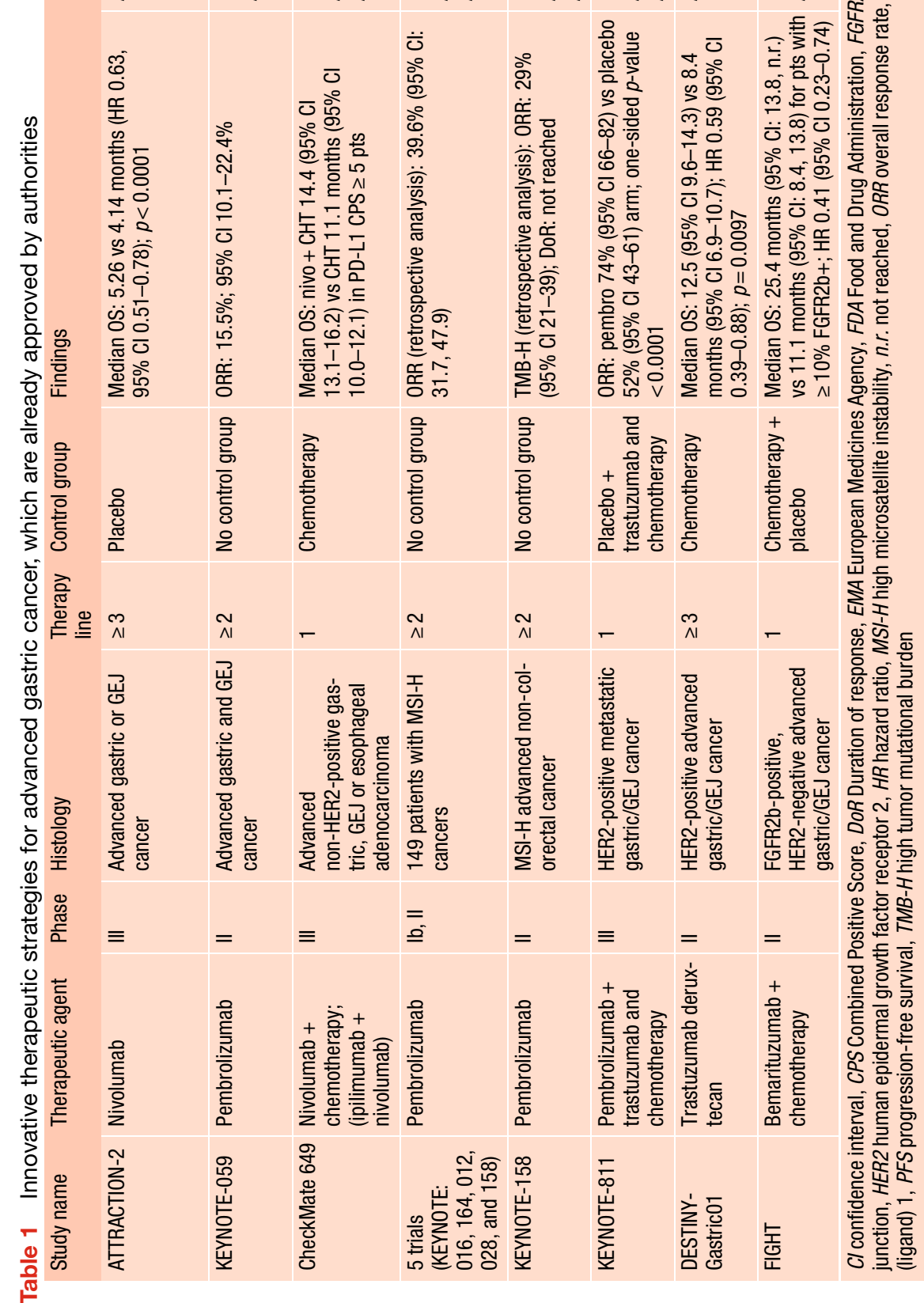


studies showed a significant benefit for all patients enrolled [4], most trials indicate that specific patient subgroups (such as high PD-L1 expression or high microsatellite instability [MSI-H]) profit most from immune checkpoint inhibition [5]. Thus, the evaluation of PD-L1 expression using Combined Positive Score (CPS) became part of the routine diagnostic work-up of tumor tissue. Yet, the cut-off levels for therapeutic approvals are still widely discussed. The results of the ATTACTION-II trial were published independent of PD-L1 expression and led to an approval of nivolumab independent of CPS expression [6].

However, in the phase II KEYNOTE-059 trial the cut-off level for PD-L1-positivity was defined as CPS $\geq 1$ (median response duration was $16.3[1.6+$ to $17.3+]$ months in patients with PD-L1-positive and 6.9 [2.4 to $7.0+]$ months and PD-L1-negative tumors) and, thus, pembrolizumab was approved by the Food and Drug Administration (FDA) as a third and further line therapy in CPS $\geq 1$ patients with metastatic gastroesophageal adenocarcinoma [5, 7]. However, by July 2021, the company voluntarily withdrew this accelerated approval indication.

However, the CheckMate 649 trial found that patients with CPS $\geq 5$ showed the most significant benefit (overall survival hazard ratio [OS HR] 0.71, 98.4\% CI $0.59-0.86 ; p<0.0001$ ) when adding nivolumab to standard chemotherapy. Interestingly, this combination was approved by the FDA in 2021 regardless of PD-L1 expression status and is currently under investigation by the European Medicines Agency (EMA) [8, 9].

Other important and already established biomarkers that indicate response to checkpoint inhibition are MSI, which is surmised to be high (MSI-H) in around $4-5 \%$ of all advanced Western gastric tumor cases, and tumor mutational burden (TMB) [10]. Thus, the FDA approved immunotherapy with pembrolizumab for the treatment of unresectable or metastatic MSI-H and TMB-H solid tumors that have progressed following prior treatment independent of tumor location and which have no satisfactory alternative treatment options ("tissue agnostic approvals") [11, 12].

These findings underline the importance of patient selection and changed the face of gastric cancer treatment.

\section{Strategies to overcome the immune cold tumors}

As mentioned above, a major issue concerning immunotherapy is that only a subset of patients achieve responses. Thus, the identification of underlying mechanisms for primary resistance to immunotherapy are of major concern [13]. Recent studies characterized these immunologically "cold" tumors by a lack of infiltrating $\mathrm{T}$ cells in the tumor microenvironment. Consequentially, tumor cells stay unrecognized by the immune system and, thus, do not respond to checkpoint inhibition.
However, it is surmised that these "cold" tumors can be transformed into "hot" and inflamed tumors by several strategies including neutralizing immunosuppression at the tumor site by combining immunotherapeutic approaches, modifying the tumor vasculature by targeting endothelial growth, targeting the tumor cells themselves with chemotherapy, inducing local inflammation with radiation therapy, or increasing the frequency of tumor-specific $\mathrm{T}$ cells with personalized approaches such as CAR T cell therapy [13].

\section{Combination with targeted therapies}

Especially, the combination with targeted therapy for angiogenesis and growth pathways has gained importance in recent years.

The combination of nivolumab and regorafenib is currently under investigation. The recently published phase Ib REGONIVO trial found encouraging antitumor activity in patients with gastric cancer in a third and further line setting (median PFS 5.6 months), thus, warranting additional investigations in larger cohorts [14]. Furthermore, ramucirumab was investigated in combination with paclitaxel plus nivolumab and showed promising anti-tumor activity (median OS 13.8 months [95\% CI 8.0-19.5 months] in $\mathrm{CPS} \geq 1$ patients) [15]. Another approach evaluated the combination treatment of lenvatinib, a multikinase inhibitor of VEGF receptors and other receptor tyrosine kinases, with pembrolizumab and a response rate of $69 \%$ (95\% CI 49-85) was demonstrated [16].

There are several ongoing trials evaluating the combination of the checkpoint inhibitor durvalumab with targeted therapies, e.g., with cabozantinib in CAMILLA trial and with raumucirumab in a phase Ib trial $[17,18]$.

\section{Combination of anti-PD-1/PD-L1 drugs with other checkpoint inhibitors}

Although the concept of neutralizing immunosuppression at the tumor site by combining immunotherapeutic approaches, the combination of anti-PD1/PD-L1 drugs with other checkpoint inhibitors, so far no practice changing trials can be reported in gastric cancer. However, several studies suggest promising new combination strategies including combination with cytotoxic T lymphocyte antigen-4 (CTLA-4; drug: ipilimumab; trials: CheckMate-032 [19], Moonlight trial [20]), lymphocyte activation gene-3 (LAG3; drug: relatlimab; trials: FRACTION [21], REACTION), T cell immunoglobulin and mucin-domain containing-3 (TIM-3), T cell immunoglobulin and ITIM domain (TIGIT), V-domain Ig suppressor of $\mathrm{T}$ cell activation (VISTA), OX40 (CD134) and B and T cell lymphocyte attenuator (BTLA) [22, 23]. 


\section{Targeted therapy for gastroesophageal tumors}

\section{Targeting HER2 beyond trastuzumab:}

In the field of HER2-positive (human epidermal growth factor receptor 2) gastroesophageal cancer, a recently published phase II trial evaluating firstline pembrolizumab and trastuzumab in combination with chemotherapy showed promising response rates and, thus, was the impulse for a randomized phase 3 clinical trial (KEYNOTE-811) [24]. First results of the KEYNOTE-811 trial found that the overall response rate (ORR) was $74 \%$ (95\% CI 66-82) in the pembrolizumab arm and $52 \%$ (95\% CI 43-61) in the placebo arm (one-sided $p$-value $<0.0001$ ) and, thus, the combination of pembrolizumab, trastuzumab and chemotherapy (ToGA regimen) has been recently approved by the FDA for the first-line treatment of HER2-positive advanced gastric cancer [25].

Another new approach that was recently approved by the FDA for HER2-positive patients who have received a prior trastuzumab-based regimen is the drug trastuzumab deruxtecan, a novel antibody-drug conjugate [26]. The drug was evaluated by the phase II DESTINY-Gastric01 trial in a third and further line setting in comparison to chemotherapy and showed a significantly longer median OS (12.5 vs. 8.4 months; HR 0.59; 95\% CI 0.39-0.88; $p=0.01$ ) [27].

\section{Attempts for novel targets}

Claudin 18.2 (CLDN18.2) protein is physiologically confined to gastric mucosa tight junctions and is exposed on the cancer cell surface upon malignant transformation.

In the phase II FAST trial, patients with advanced gastric cancer and a CLDN18.2 expression in $\geq 70 \%$ of tumor cells were treated with the anti-Claudin 18.2 antibody zolbetuximab in combination with chemotherapy. Zolbetuximab generated prolonged OS rates in this patient subgroup (HR 0.55; 95\% CI 0.39-0.77; $p<0.0005$ ) [28]. Based on this data, two phase III trials have been initiated, which will reveal first results within the next few years.

Fibroblast growth factor receptor $2 b$ (FGFR2b) is another very interesting target, which was tested in the FIGHT study. The anti-FGFR2b antibody bemarituzumab in combination with chemotherapy as firstline revealed very promising results in FGFR2b-positive patients, which led to the designation of bemarituzumab as a breakthrough therapy by the FDA (OS HR 0.66; 95\% CI 0.39-1.12) [29, 30].

\section{Discussion}

Despite the improvement of modern cancer medicine including immunotherapies and targeted therapies, new therapeutic approaches seem to be efficiently in only specific subgroups of patients. To improve pa- tient outcome with these new treatment options, it is of highest importance to define these subgroups more accurately. The evaluation and implementation of new biomarkers seems to be the key for adequate patient selection leading to high treatment efficacy. In case of immunotherapy, this selection is mainly based on PD-L1 expression, MSI status and, more recently, on TMB. However, looking at response rates it is evident that despite these biomarkers there is still significant percentage of patients who do not respond to treatment. Thus, underlining the fact that further strategies should be implemented to develop predictive markers.

Another critical issue is to overcome so called "cold" tumors, thereby improving treatment response. New combination strategies including combinations of the inhibition of the PD-(L) 1 axis with chemotherapy, radiation therapy, other immune checkpoint inhibitors and targeted therapies are underway into clinical practice to overcome this treatment resistance.

Finally, critical issues to consider for new drug approvals are tolerability, impact on quality of life, and financial considerations. Future studies need to include these major considerations in clinical trial design in order to achieve more adequate implementation of new therapeutic agents into real-life cohorts and guidelines.

In conclusion, recently published studies led to a paradigm change of advanced gastric cancer treatment and several new innovative approaches are underway to further improve the management of patient subgroups.

\section{Take home message}

New innovative targets and drugs are underway to improve the care of patients with advanced gastric cancer. This mini-review highlights promising new treatment approaches and their clinical relevance.

Funding The authors gratefully acknowledge Medical University of Vienna's core funding to the Department of Medicine I.

Funding Open access funding provided by Medical University of Vienna.

Conflict of interest H.C. Puhr has received travel support from Eli Lilly, MSD, Novartis, Pfizer and Roche. A. IlhanMutlu participated in advisory boards from MSD, BMS and Servier, received lecture honoraria from Eli Lilly, MSD, BMS and Servier, is the local PI for clinical trials sponsored by BMS and Roche and received travel support from BMS, Roche, Eli Lilly and Daiichi Sankyo.

Open Access This article is licensed under a Creative Commons Attribution 4.0 International License, which permits use, sharing, adaptation, distribution and reproduction in any medium or format, as long as you give appropriate credit to the original author(s) and the source, provide a link to the Creative Commons licence, and indicate if changes were made. The images or other third party material in this article are included in the article's Creative Commons licence, unless 
indicated otherwise in a credit line to the material. If material is not included in the article's Creative Commons licence and your intended use is not permitted by statutory regulation or exceeds the permitted use, you will need to obtain permission directly from the copyright holder. To view a copy of this licence, visit http://creativecommons.org/licenses/by/4.0/.

\section{References}

1. Ferlay J, Colombet M, Soerjomataram I, Mathers C, Parkin DM, Piñeros M, et al. Estimating the global cancer incidence and mortality in 2018: GLOBOCAN sources and methods. Int JCancer. 2019;144(8):1941-53.

2. Vrána D, Matzenauer M, Neoral Č, Aujeský R, Vrba R, Melichar B, etal. From tumor immunology to immunotherapy in gastric and esophageal cancer. Int J Mol Sci. 2018;20(1):13.

3. LordickF, Shitara K, Janjigian YY. New agents on the horizon in gastric cancer. Ann Oncol. 2017;28(8):1767-75.

4. Kang Y-K, Boku N, Satoh T, Ryu M-H, Chao Y, Kato K, et al. Nivolumab in patients with advanced gastric or gastro-oesophageal junction cancer refractory to, or intolerant of, at least two previous chemotherapy regimens (ONO-4538-12, ATTRACTION-2): a randomised, double-blind, placebocontrolled, phase 3 trial. Lancet. 2017;390(10111):2461-71.

5. Fuchs CS, Doi T, Jang RW, Muro K, Satoh T, Machado M, et al. Safety and efficacy of pembrolizumab monotherapy in patients with previously treated advanced gastric and gastroesophageal junction cancer: phase 2 clinical KEYNOTE059 trial. JAMAOncol. 2018;4(5):e180013.

6. Chen L-T, Satoh T, Ryu M-H, Chao Y, Kato K, Chung HC, et al. A phase 3 study of nivolumab in previously treated advanced gastric or gastroesophageal junction cancer (ATTRACTION-2): 2-year update data. Gastric Cancer. 2020;23(3):510-9.

7. Food and Drug Administration. FDA grants accelerated approval to pembrolizumab for advanced gastric cancer. 2017. https://www.fda.gov/drugs/resourcesinformation-approved-drugs/fda-grants-acceleratedapproval-pembrolizumab-advanced-gastric-cancer. Accessed 01 July 2021.

8. Janjigian YY, Shitara K, Moehler M, Garrido M, Salman P, Shen L, et al. First-line nivolumab plus chemotherapy versus chemotherapy alone for advanced gastric, gastrooesophageal junction, and oesophageal adenocarcinoma (CheckMate 649): a randomised, open-label, phase 3 trial. Lancet. 2021;398(10294):27-40.

9. FoodandDrugAdministration. FDAapprovesnivolumabin combination with chemotherapyformetastaticgastric cancer and esophageal adenocarcinoma. 2021. https://www. fda.gov/drugs/resources-information-approved-drugs/ fda-approves-nivolumab-combination-chemotherapymetastatic-gastric-cancer-and-esophageal. Accessed 01 July 2021.

10. Polom K, Marano L, Marrelli D, De Luca R, Roviello G, Savelli V, et al. Meta-analysis of microsatellite instability in relation to clinicopathological characteristics and overall survival in gastric cancer. Br JSurg. 2018;105(3):159-67.

11. Food and Drug Administration. FDA approves pembrolizumab for adults and children with TMB-H solid tumors. 2020. https://www.fda.gov/drugs/drug-approvalsand-databases/fda-approves-pembrolizumab-adultsand-children-tmb-h-solid-tumors. Accessed 01 July 2021.

12. Food and Drug Administration. FDA grants accelerated approval to pembrolizumab for first tis- sue/site agnostic indication. 2017. https://www. fda.gov/drugs/resources-information-approved-drugs/ fda-grants-accelerated-approval-pembrolizumab-firsttissuesite-agnostic-indication. Accessed 01 July 2021.

13. Ochoa de Olza M, Navarro Rodrigo B, Zimmermann S, Coukos G. Turning up the heat on non-immunoreactive tumours: opportunities for clinical development. Lancet Oncol. 2020;21(9):e419-e30.

14. Fukuoka S, Hara H, Takahashi N, Kojima T, Kawazoe A, Asayama M, et al. Regorafenib plus nivolumab in patients with advanced gastric or colorectal cancer: an open-label, dose-escalation, and dose-expansion phase Ib trial (REGONIVO, EPOC1603). J Clin Oncol. 2020;38(18):2053-61.

15. Hironaka S, Kadowaki S, Izawa N, Nishina T, Yamanaka T, Minashi K, et al. A phase I/II study of nivolumab, paclitaxel, and ramucirumab as second-line in advanced gastric cancer. JClin Oncol. 2020;38(4_suppl):352.

16. Kawazoe A, Fukuoka S, Nakamura Y, Kuboki Y, Wakabayashi M, Nomura S, et al. Lenvatinib plus pembrolizumab in patients with advanced gastric cancer in the first-line or second-line setting (EPOC1706): an open-label, single-arm, phase 2 trial. Lancet Oncol. 2020;21(8):1057-65.

17. Saeed A, Koestler D, Williamson SK, Baranda JC, Sun W, Al-Rajabi RMT, et al. A phase Ib trial of cabozantinib in combination with durvalumab (MEDI4736) in previously treated patients with advanced gastroesophageal cancer and other gastrointestinal (GI) malignancies (CAMILLA). JClin Oncol. 2019;37(8_suppl):TPS56.

18. Bang Y-J, Golan T, Lin C-C, Dahan L, Fu S, Moreno V, et al. Ramucirumab (Ram) and durvalumab (Durva) treatment of metastatic non-small cell lung cancer (NSCLC), gastric/gastroesophageal junction (G/GEJ) adenocarcinoma, and hepatocellular carcinoma (HCC) following progression on systemic treatment(s). J Clin Oncol. 2019;37(15_suppl):2528.

19. Janjigian YY, Bendell J, Calvo E, Kim JW, Ascierto PA, Sharma P, et al. CheckMate-032 study: efficacy and safety of nivolumab and nivolumab plus ipilimumab in patients with metastatic esophagogastric cancer. JClin Oncol. 2018;36(28):2836-44.

20. Al-Batran S-E, Pauligk C, Goetze TO, Riera-Knorrenschild J, Goekkurt E, Angermeier S, et al. Modified FOLFOX versus modified FOLFOX plus nivolumab and ipilimumab in patients with previously untreated advanced or metastatic adenocarcinoma of the stomach or gastroesophageal junction: Moonlight, a randomized phase 2 trial of the German Gastric Group of the AIO. J Clin Oncol. 2019;37(15_suppl):TPS4144.

21. Aanur P, Gutierrez M, Kelly RJ, AjaniJA, Ku GY, Denlinger CS, et al. FRACTION (Fast Real-time Assessment of Combination Therapies in Immuno-Oncology)-gastric cancer (GC): a randomized, open-label, adaptive, phase 2 study of nivolumab in combination with other immuno-oncology (IO) agents in patients with advanced GC. J Clin Oncol. 2017;35(15_suppl):TPS4137.

22. Qin S, Xu L, Yi M, Yu S, Wu K, Luo S. Novel immune checkpoint targets: moving beyond PD-1 and CTLA-4. Mol Cancer. 2019;18(1):155.

23. Alves Costa SC, Facchinetti F, Routy B, Derosa L. New pathways in immune stimulation: targeting OX40. ESMO Open. 2020;5(1):e573.

24. Janjigian YY, Maron SB, Chatila WK, Millang B, Chavan SS, Alterman C, et al. First-line pembrolizumab and trastuzumab in HER2-positive oesophageal, gastric, or gas- 


\section{short review}

tro-oesophageal junction cancer: an open-label, singlearm, phase 2 trial. Lancet Oncol. 2020;21(6):821-31.

25. Food and Drug Administration. FDA grants accelerated approval to pembrolizumab for HER2-positive gastric cancer. 2021. https://www.fda.gov/drugs/resourcesinformation-approved-drugs/fda-grants-acceleratedapproval-pembrolizumab-her2-positive-gastric-cancer. Accessed 01 July 2021.

26. Food and Drug Administration. FDA approves famtrastuzumab deruxtecan-nxki for HER2-positive gastric adenocarcinomas. 2021. https://www.fda.gov/drugs/ resources-information-approved-drugs/fda-approvesfam-trastuzumab-deruxtecan-nxki-her2-positive-gastricadenocarcinomas. Accessed 01 July 2021.

27. Shitara K, Bang Y-J, Iwasa S, Sugimoto N, Ryu M-H, Sakai D, et al. Trastuzumab deruxtecan in previously treated HER2positive gastric cancer. NEnglJ Med. 2020;382(25):2419-30.

28. Sahin U, Türeci Ö, Manikhas G, Lordick F, Rusyn A, Vynnychenko I, et al. FAST: a randomised phase II study of zolbetuximab (IMAB362) plus EOX versus EOX alone for first-line treatment of advanced CLDN18.2-positive gastric and gastro-oesophageal adenocarcinoma. Ann Oncol. 2021;32(5):609-19.

29. Catenacci DVT, Kang Y-K, Saeed A, Yamaguchi K, Qin S, Lee K-W, et al. FIGHT: a randomized, double-blind, placebo-controlled, phase II study of bemarituzumab (bema) combined with modified FOLFOX6 in 1L FGFR2b+ advanced gastric/gastroesophageal junction adenocarcinoma (GC).JClin Oncol.2021;39(15_suppl):4010.

30. ASCO Post Staff. FDA pipeline: bemarituzumab granted breakthrough therapy designation for FGFR2b-overexpressing, HER2-negative gastric cancer. 2021. https:// ascopost.com/news/april-2021/fda-pipeline-bemarituzu mab-granted-breakthrough-therapy-designation-for-fgfr 2b-overexpressing-her2-negative-gastric-cancer/. Accessed 01 July 2021.

Publisher's Note Springer Nature remains neutral with regard to jurisdictional claims in published maps and institutional affiliations.

- For latest news from international oncology congresses see: http://www.springermedizin.at/ memo-inoncology 